\title{
Anti-fibrotic agents could be the game changer for Post-COVID Pulmonary Fibrosis treatment - A mini review
}

\author{
Pallab Chakraborty $^{1}$, Kaustav Chakraborty ${ }^{2 *}$ \\ ${ }^{1}$ Post Graduate student; University of Calcutta, West Bengal, India \\ ${ }^{2}$ Department of Zoology, S.B.S. Government College, Hili, West Bengal, India \\ *Corresponding Author: KaustavChakraborty, PhD, Assistant Professor (W.B.E.S), \\ Department of Zoology, S.B.S. Government College, Hili, Dakshin Dinajpur - 733126, West \\ Bengal, India. Email Id: kaustavc17@gmail.com , Contact No: +91-9836856131
}

\begin{abstract}
:
Total 219 countries and territories globally suffering from the recent pandemic COVID-19 is now in its second wave with more brutality, caused by severe acute respiratory syndrome coronavirus 2 (SARS-CoV-2) . It has several symptoms like as persistent fever; respiratory illnesses; cough; fatigue; shortness of breath; loss of appetite; persistent pain or pressure in the chest; dysgeusia; acute respiratory distress syndrome (ARDS) etc., and here the things to worry about is the development of pulmonary fibrosis after COVID-19 in both peoples who had died of due to acute respiratory distress syndrome (ARDS) or those who survived. Due to COVID-19, dysregulated immune response and wound repair mainly in elderly patients causes this secondary pulmonary fibrosis. Thus using anti-fibrotic agents could be meaningful in these circumstances although their efficacy in treating COVID-19 is subject to more detailed laboratory research works. In this review article you will get to know about the lung fibrosis generation due to COVID-19 infection, about anti-fibrotic agents and the currents challenges of this field.
\end{abstract}

Key words: COVID-19; post-COVID pulmonary fibrosis; lung injury, anti-fibrotic agents.

\section{Abbreviation:}

Severe acute respiratory syndrome coronavirus 2 (SARS-CoV-2), acute respiratory distress syndrome (ARDS), Middle East Respiratory Syndrome (MERS), severe acute respiratory 
syndrome (SARS), ORF (open reading frame), dipeptidyl peptidase 4 (DPP4), angiotensinconverting enzyme 2 (ACE2), transforming growth factor-beta 1 (TGF- $\beta 1$ ), angiotensinogen (AGT), connective tissue growth factor (CTGF), vascular endothelial growth factor (VEGF), fibronectin (FN), interleukin-6 (IL-6), 3C-like protease (3CLpro), RNA-dependent RNA polymerase (RdRp), papain-like cysteine protease (PLpro), idiopathic pulmonary fibrosis (IPF), chronic obstructive pulmonary disease (COPD), Mesenchymal Stem Cells (MSCs), Galectin-3 (gal-3), Hepatocyte growth factor (HGF).

\section{Introduction:}

The severe acute respiratory syndrome coronavirus 2 (SARS-CoV-2) that is earlier known as 2019 novel coronavirus (2019-nCoV) causes COVID-19 viral disease globally and till now affected 219 countries [1-5]. Due to this outbreak and pandemic situation 155,844,806 cases that is 155(M) million and 3,255,604 deaths reported globally as of May 06, 2021, 05:26 GMT and in India the death number is $226 \mathrm{~K}$ and the current cases distribution of COVID-19 also represented here in figure $1[6,7]$.

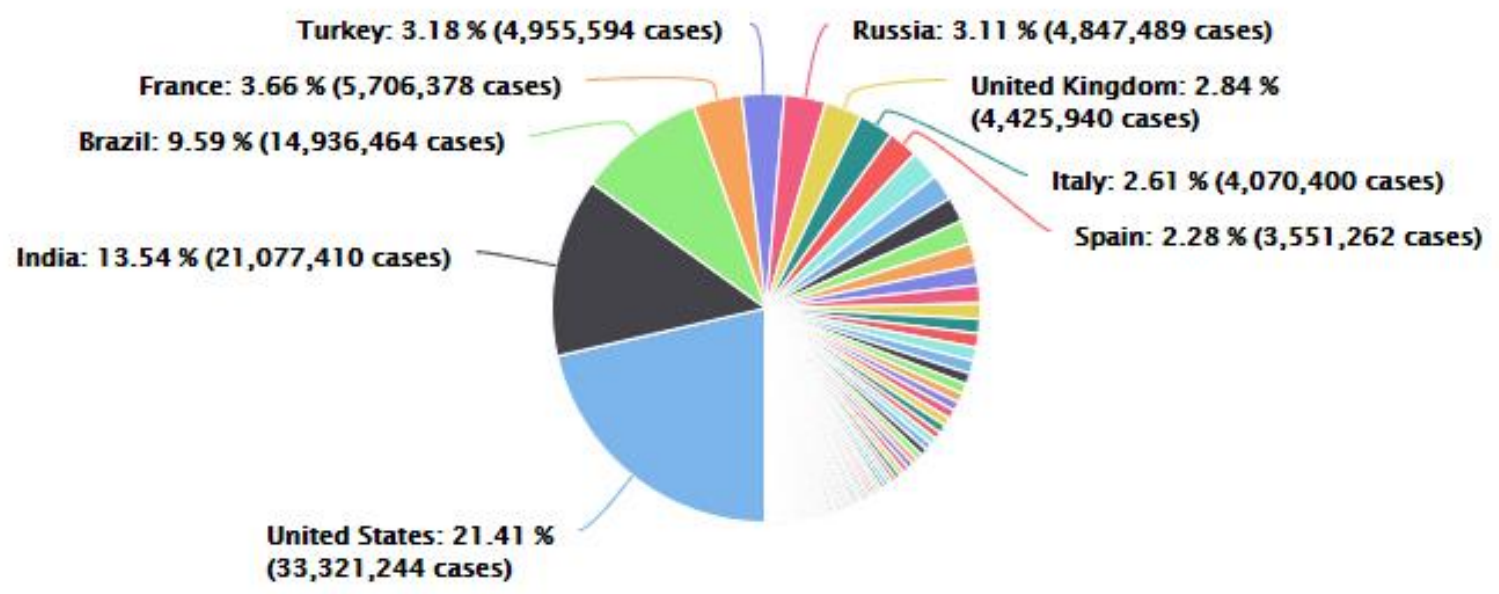

Figure 1 Countries cases distribution of COVID-19 infectionas of May 06, 2021, 05:26 GMT (source:Worldometer - www.worldometers.info[7] 
Ortho-coronavirinae is the subfamily of this virus where the Alpha, Beta, Gamma and Delta coronavirus are four genera of this subfamily and other three [5,8] are Middle East Respiratory Syndrome, or MERS, severe acute respiratory syndrome, or SARS and finally SARS-CoV-2 , the novel COVID-19[1,9]. In December 2019, this virus first reported in Wuhan City, Hubei Province, Central China, that causing some common symptoms like fever, dry cough and fatigue on the other some severe symptoms like as respiratory illnesses, shortness of breath, loss of appetite, persistent pain or pressure in the chest, high temperature (above $38{ }^{\circ} \mathrm{C}$ ) and persistent fever, dysgeusia, lung injury acute respiratory distress syndrome (ARDS) with epithelial and endothelial injury in some individuals[10-12]. It is reported that among the seventh members, four result in minor symptoms related to the upper respiratory tract. On the other hand, the rest three including SARS-CoV-2 cause lower respiratory tract infections that's leads to major lung complications such as acute respiratory distress syndrome (ARDS), cytokine release syndrome and pulmonary fibrosis (PF) which starts early in the course of ARDS as PF is a recognized sequel of ARDS [10,13]. The most dangerous consequences of this viral infection that was already hypothesized $[14,15]$ is found to be real in the current scenario as of chest physicians from all over the world recognized peoples recovered from COVID-19 still left with lung fibrosis that is termed here as post-COVID lung fibrosis $[13,16]$. It has been suggested that the pulmonary fibrosis is interconnected to the pathology of ARDS, that's has three phases: exudative, proliferative, and fibrotic, where in the first week or you can say at initial stage, the diffuse alveolar damage, the exudative phase with edema, hyaline membranes, and interstitial acute inflammation occurs and next to that an organizing phase with loose organizing fibrosis and fibro-proliferative phase and, in non-survivors, end-stage fibrotic lung can be seen[11,13,16,17].In COVID-19 disease, the various pro-inflammatory cytokines are produced abnormally in higher level along with excessive infiltration of inflammatory cells, the phenomenon is also known as 'cytokine strom', which is believed to be key event in COVID-19 mortality and morbidity and due to this abnormal inflammatory event, the pulmonary fibrosis can be promoted $[13,18]$. Reported data also supports the view that about $40 \%$ of patients with COVID-19 develop ARDS, and 20\% experienced more severity and may further leads to fibrosis later $[13,17]$.

For this reason using anti-fibrotic therapies could be a game changer in this situation and some of the approved anti-fibrotic drugs for e.g. pirfenidone and nintedanib [19,20] which are effective 
against lung functional abnormalities and improving life can be administrated. Here in this article, we will discuss about the post-COVID-19 lung fibrosis and how this lung fibrosis gets developed and disrupted by COVID-19virus.After that we have discussed about some potential anti-fibrotic agents that could be used as a potential therapeutics for post-COVID pulmonary fibrotic patients depending on other co-morbidities and severity of the disease. Therefore, we have listed some open questions which are the limitations and future challenges of this field.

\section{Coronavirus disease 2019 (COVID-19) pathology:}

The severe acute respiratory syndrome coronavirus 2 (SARS-CoV-2) is a RNA virus and its genome size is $30 \mathrm{~kb}$, that's mainly code for several proteins (poly proteins) also known as ORF1a/b (open reading frame) and it furthers cleaved into more several proteins parts among them, 5 accessory and 4 structural proteins important for its assembly and infectiveness including spike surface glycoprotein, membrane protein, envelop protein and neucleocapsid $[1,21,22]$. It is the seventh member from the family that infect humans with approximately $70 \%$ genetic similarity to SARS-CoV and the site of infection is mainly depends upon the presence of the dipeptidyl peptidase 4 (DPP4) and angiotensin-converting enzyme 2 (ACE2), where the viral spike protein binds also similar for MERS and SARS [1,10,23-25]. As most of the human cells related to lower respiratory tract including endothelial, alveolar cells, further tracheal, bronchial cells bearing the ACE2 receptor, the SARS-CoV-2 virus which has 10-20 fold greater affinity towards this than SARS-CoV easily in a faster way enters and upon replicating inside host cells initiates the immune-pathogenesis and resulting huge amount of local cytokines secretion causing lung tissue damages, acute respiratory distress syndrome (ARDS), multiple organ failure[1,26,27].Decreased immune functions, reduction of lymphocytic T Cells (CD4+ and CD8+) and natural killer (NK) cells and the high level of inflammatory cytokines (IL-2, IL-6, IL7, IL-10), MCP-1, MIP-1A and TNF- $\alpha$ are linked with SARS-CoV-2 severity [28,29]. Most susceptible groups for this viral infection are older peoples but in the second wave in India from $18+$ young generation are included in this range, even kids (below the age of 16) are right now also prone to the dangers [30]. 


\section{Pulmonary fibrosis after COVID-19:}

Several data available from autopsy, clinical radiography have shown that the novel corona virus damages mainly the respiratory system and in the long run its causes pulmonary fibrosis $[10,11,13,17]$. We know very well about the pathogenic mechanism of viral lung fibrosis, as it has been studied with others viruses like influenza and SARS, where it has been reported that, in case of H1N1 influenza the transforming growth factor-beta 1 (TGF- $\beta 1$ ) level increased, found similar for SARS-CoV-1 (outbreak in 2002) and due to higher level of this cytokine the extracellular matrix proteins deposition and fibroblast differentiations takes place in abnormal manners that finally promote the lung fibrosis $[16,31,32]$. In case of novel corona virus, the molecular mechanism for the progression of pulmonary fibrosis is not clearly understood but believed to depends upon various factors and similar to SARS-CoV [13].A very recent research by Xu et al., (2020) confirmed that SARS-CoV-2 binds to the ACE2 caused activation of fibrosis related process and genes, like as altered expression in mRNA and protein level of angiotensinogen (AGT), transformation growth factor-beta 1 (TGF- $\beta 1$ ), connective tissue growth factor (CTGF), vascular endothelial growth factor (VEGF) and fibronectin (FN), are observed by the bioinformatics studies which are also found in the patients with lung fibrosis [29]. So here in SARS-CoV-2, it may be also possible that its first activates the TGF- $\beta$ pathway then through this signaling its increased the ECM proteins mainly the FN and the thus the alveolar-epithelial cells may result in lung fibrosis [16,33]. In addition to this, decreasing of ACE-2 level causes upregulation of angiotensin 2, which plays key role in the development of inflammation and fibrosis by generation of reactive oxygen species(ROS), production of the pro-inflammatory cytokines mainly interleukin-6 (IL-6) and IL-8 and activation of TGF- $\beta$ signaling is also postulated [16,34]. Furthermore, It is also possible that oxygen toxicity (oxygen-derived free radicals) and mechanical stress or ventilation (including barotrauma and volutrauma) can cause lung and pulmonary injury, contributing to ARDS in which during inflammatory stage matrix metallo-protease released in abnormal manners and causes endothelial injury, increase cytokine production, upsurge epithelial-mesenchymal transition (EMT) and collagen deposition in lung and finally all these contributing to pulmonary fibrosis [16,35-37].

From several scientific reports, medical observations and evidences we can summarize that after SARS-CoV-2 infection in some patients, activation of the TGF- $\beta$ signaling; 
inflammation(cytokine storm);ARDS pathology the well-known acute and diffuse inflammatory damage into the alveolar-capillary barrier; high ROS level; accumulation and deposition of ECM proteins all are involved for the post-COVID lung fibrosis generation. There may be more unknown pathways, signaling crosstalk's which are directly or indirectly causes lung fibrosis are yet to discover and gradually will be published through research in upcoming days.

\section{Anti-fibrotics, the game changer:}

Based on current scenario, using anti-fibrotic drugs are may be very useful to tackle the postCOVID pulmonary fibrosis $[11,17,38]$. There are some well-known chemical compounds such as pirfenidone and nintedanib (FDA-approved) and some natural compounds including quercetin, baicalin and baicalein, and salvianolic acid B are recognized as potential anti-fibrotic agents that can be applied to the COVID-19 patients to save them from the development of pulmonary fibrosis and other consequences. The chemical structures of these potential drugs are represented here in the figure 2. Pirfenidone is an oral drug that has anti-fibrotic, anti-oxidative and antiinflammatory properties. Nintedanib is also an good anti-fibrotic agents that's being an tyrosine kinase inhibitor regulate downstream signaling of fibrosis, it also has anti-inflammatory property and inhibits IL-6 and IL-1[11,38,39]. Others well-known natural compounds that exhibit antifibrotic effects are some flavonoids and non-flavonoids. 


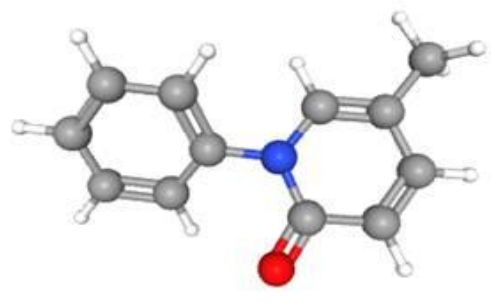

Pirfenidone

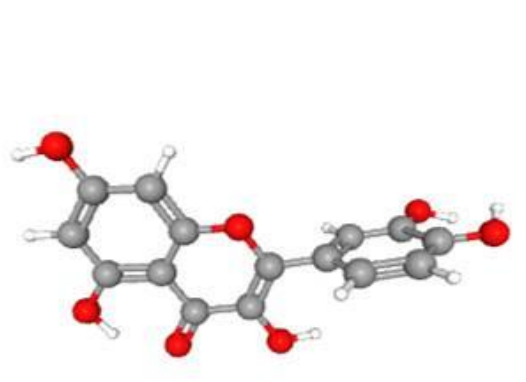

Quercetin

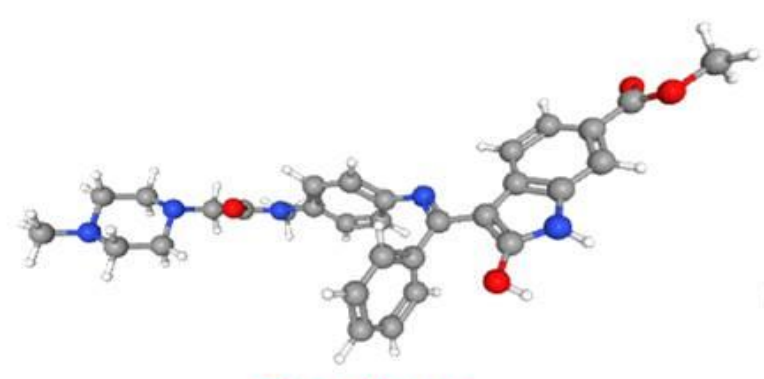

Nintedanib

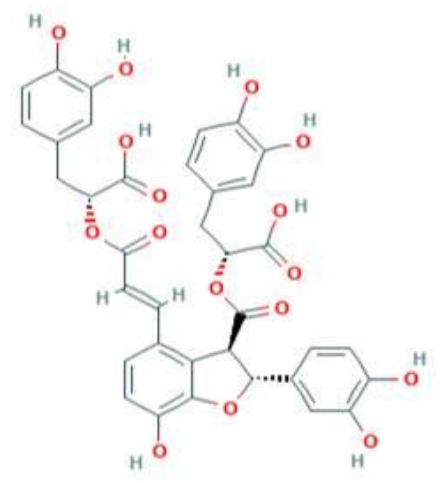

Salvianolic acid B

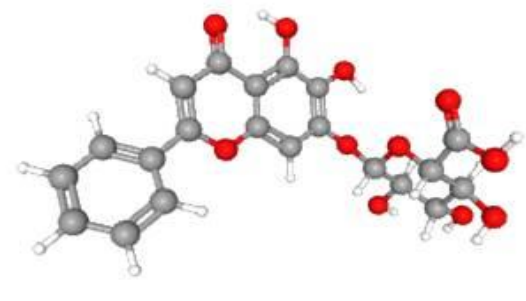

Baicalin

Structure source: https://pubchem.ncbi.nlm.nih.gov/

Figure 2: Chemical structure of some potential anti fibrotic compounds.

From fruits and vegetables we have the quercetin which shows dose dependent anti-fibrotic activity in vitro [40]. Recent in silico and in vitro studies suggested that quercetin can performed key role to modulate various stages of the coronavirus entry into host cells and also with replication cycle [40,41]. Previous studies with viral 3C-like protease (3CLpro) of SARS-CoV and with others important targets parts of SARS-CoV-2 like the host entry apparatus, Spike protein and ACE2 receptor, the RNA-dependent RNA polymerase (RdRp), that is crucial for the replication of viral RNA, and papain-like cysteine protease (PLpro), that's controlling virus maturation, impairment of host inflammation, suggested that the quercetin binds better with all these[42]. Furthermore, The baicalin and baicalein also can be acts as novel inhibitors of SARSCoV-2 3CL protease [43]. The salvianolic acid B that's is available in Salvia miltiorrhiza, it is also found effective to inhibit the entry of 2019-nCoV spike pseudovirus into angiotensinconverting enzyme 2 high-expressing HEK293T cells (ACE2h) cells by binding to the RBD of 
the 2019-nCoV spike protein and ACE2 protein [40,44]. The full list of these chemical and herbal compounds and their key functions including antiviral role summarized in the table 1.

Table 1: Brief details about some well-known anti-fibrotic drugs.

\begin{tabular}{|c|c|c|c|}
\hline $\begin{array}{c}\text { Drugs/ } \\
\text { Compounds }\end{array}$ & Key function & Antiviral activity & References \\
\hline Pirfenidone & $\begin{array}{l}\text { It has anti-fibrotic, anti-inflammatory, } \\
\text { Anti-oxidative, inhibits IL-6 and IL-1 }\end{array}$ & Not reported & {$[11,38]$} \\
\hline Nintedanib & $\begin{array}{c}\text { It inhibits downstream molecules } \\
\text { involved in fibrosis, control fibroblast } \\
\text { differentiations }\end{array}$ & Not reported & {$[11,38,39]$} \\
\hline Quercetin & $\begin{array}{l}\text { In vitro study shown dose-dependent } \\
\text { anti-fibrotic activity and low cytotoxicity }\end{array}$ & $\begin{array}{l}\text { Anti-COVID-19 activity by modulating } \\
\text { its entry into host cells and also with } \\
\text { replication cycle }\end{array}$ & {$[40,41]$} \\
\hline $\begin{array}{l}\text { Baicalin and } \\
\text { Baicalein }\end{array}$ & $\begin{array}{l}\text { Anti-inflammation, anti-fibrotic } \\
\text { inflammation and anti -fibrosis }\end{array}$ & $\begin{array}{c}\text { Antiviral property against influenza and } \\
\text { dengue virus also Anti COVID-19 } \\
\text { activity }\end{array}$ & {$[43,45,46]$} \\
\hline $\begin{array}{l}\text { Salvianolic } \\
\text { acid B }\end{array}$ & Anti-fibrosis, Suppressed TGF- $\beta 1$. & $\begin{array}{c}\text { Anti COVID-19 activity by inhibiting the } \\
\text { entry of 2019-nCoV spike pseudovirus } \\
\text { into ACE2 cells. }\end{array}$ & {$[40,44]$} \\
\hline
\end{tabular}

\section{Discussion:}

Now we have some ideas regarding development and progression of fibrosis in lungs of some COVID-19 patients and how the pathology of lung injury gets modulated by the COVID-19 viral infection. In this article we have summarized some potent anti-fibrotic agents both chemical and herbal that could be used for better treatment. Upon analysis the data represented here in table 1 and through literatures survey $[40,41,43,44,46]$, we have found that natural compounds including quercetin, baicalin and baicalein, and salvianolic acid B could be serve as most potential agents for the treatment of post-COVID lung fibrosis as they possess both antiviral and anti-fibrotic properties. Thus using anti-fibrotic agents could be the game changer. According to George PM et al., (2020),[11,16] using the anti-fibrotics can be considered within the first week of ARDS 
onset but their efficacy in treating COVID-19 is subject to more detailed laboratory research. The anti-fibrotics agents also have some pleotropic effects so how to tackle this problem should be our prior concerns. A randomized, open clinical trial to evaluate the efficacy and safety of pirfenidone (ClinicalTrials.gov identifier:NCT04282902) is currently going on and in its phase 3 and more longer follow-up durations are mandatory to get better inference [15]. For the natural compounds the low bioavailability and lacking of proper dose standardization is a limitation. Instead of these we can think of using them for the better treatment purpose in this emergency.

Apart from these anti-fibrotic approaches several other therapeutic strategies may be useful in this field. A group of researchers performed bioinformatics studies where they found that the idiopathic pulmonary fibrosis (IPF), chronic obstructive pulmonary disease (COPD) patients are subject to high risk to be infected by SARS-CoV-2. The transcriptomic analysis also revealed similar pathways and identified some genes which are responsible for diffident types of respiratory diseases like IPF and COPD, so that could be used for better therapeutic targets [47]. On the other, using Mesenchymal Stem Cells (MSCs) as therapeutics also could be a very important approach that has been reviewed by Vishnupriya et al., (2021) [10]. The MSCs, reached the site of injury and inhibits the inflammations, its secretes some factors like hepatocyte growth factor (HGF), which disturbed the TGF- $\beta$ signaling and prevent tissue fibrosis [10]. The Galectin-3 (gal-3) that's a carbohydrate binding protein expressed by the macrophages and alveolar epithelial cells of lung and found to be related with the abnormal inflammation known as 'cytokine strom' and lung fibrosis so using inhibitors for them could also be a good therapeutic approach that has been reviewed by Garcia-Revilla et al., 2020 [18]. Recent study by McGroder et al., (2021) suggested that age-adjusted telomere length should be considered as an independent risk factor for post-COVID-19 pulmonary fibrosis [48]. Proper identification of risk factors and biomarkers for earlier stage of lung fibrosis is very crucial to diagnose which of these patients will proceed to develop fibrosis and so that we can implement specific therapeutics.

Although, for now it is still a big question that why only some individuals develop this postCOVID pulmonary fibrosis and others recovers from it. Probably there are involvements of some other risk factors such as co-morbidities, age, severity of initial illness, and duration of mechanical ventilation, several genetic or epigenetic factors which are also a subject of research. 
There are some other obvious questions that are listed as open questions are needed to be resolved in the upcoming days.

\section{Open Questions}

1. What are the multi factors related to this post-COVID pulmonary fibrosis development?

2. Can we use the anti-fibrotics as a combinational therapy with others antiviral and antiinflammatory drugs?

3. What will be the efficacy of anti-fibrotics within vaccinated and non-vaccinated persons, among peoples who have other co-morbidities?

4. What is the underlying mechanism for activation of TGF- $\beta$ signaling which is regulated by SARS-CoV-2 in some COVID-19 patients or there is involvement of any other signaling cross-talk?

\section{Conclusion:}

Finally after considering all the above-mentioned points, now we can say that it will be very meaningful to use the anti-fibrotic drugs against post-COVID pulmonary fibrosis in future. Although better understanding of pathophysiology of this lung fibrosis is required for better inference. In case of personalized gene based treatment, more knowledge about the confirmed risk factors responsible is required. In this situation detailed laboratory work to be performed to fill the gaps.

\section{Author's contributions:}

The research idea came from KC. PC did literature review, prepared the manuscript and performed referencing under the supervision of $\mathrm{KC}$. $\mathrm{KC}$ critically revised the work. All authors subsequently revised the manuscript and then approved.

\section{Declaration:}


Information's presented here after giving proper credits to the original authors or source. We further make no representations that the data available in the referenced papers is free from error. We apologize if we missed to cite your valuable paper due to place issue. The graphical representation (fig.1) collected from the Worldometer - www.worldometers.info.

Acknowledgement: Authors are supported by their institute.

Conflict of Interest: The author(s) have declared they have no conflict of interest

Ethical approval: Not required

Funding: No funding

\section{References:}

1. Li H, Liu S, Yu X, Tang S, Tang C. Coronavirus disease 2019 (COVID-19): current status and future perspectives. Int J Antimicrob Agents 2020; 5:105951.

2. Xu X, Chen P, Wang J, Feng J, Zhou H, Li X et al. Evolution of the novel coronavirus from the ongoing Wuhan outbreak and modeling of its spike protein for risk of human transmission. Sci China Life Sci 2020; 63:457-60.

3. Wong JEL, Leo YS TC. COVID-19 in Singapore-current experience: critical global issues that require attention and action. JAMA 2020; .

4. Li Q, Guan X, Wu P, Wang X, Zhou L, Tong Y et al. Early transmission dynamics in Wuhan, China, of novel coronavirus-infected pneumonia. N Engl J Med 2020; 382:1199_ 1207.

5. Kaustav Chakraborty. COVID-19: Zoonotic Origin, Interspecies Transmission, VirusHost Interaction and Animals Susceptibility to SARS-CoV-2”. EC Pulmonol Respir Med 2020; 9:52-60.

6. https://en.wikipedia.org/wiki Template:COVID-19_pandemic_data. no date; .

7. https://www.worldometers.info/coronavirus/coronavirus-death-rate/. no date; .

8. https://www.cdc.gov/coronavirus/types.html. no date; .

9. $\quad$ Banerjee A, Kulcsar K, Misra V, Frieman M MK. Bats and coronaviruses. Viruses. 2019;

10. Vishnupriya M, Naveenkumar M, Manjima K, Sooryasree N V., Saranya T, Ramya S, Winster SH, Paulpandi M, Balachandar V, Arul N. Post-COVID pulmonary fibrosis: 
Therapeutic efficacy using with mesenchymal stem cells - How the lung heals. Eur Rev Med Pharmacol Sci 2021; 25:2748-2751.

11. George PM, Wells AU, Jenkins RG. Personal View Pulmonary fibrosis and COVID-19 : the potential role for antifibrotic therapy. Lancet Respir 2020; 8:807-815.

12. https://www.who.int/emergencies/diseases/novel-coronavirus-2019/advice-for-public. no date; .

13. Vasarmidi E, Tsitoura E, Spandidos DA, Tzanakis N, Antoniou KM. Pulmonary fibrosis in the aftermath of the C OVID-19 era ( Review ). 2020; 2557-2560.

14. Grillo F, Barisione E, Ball L, Mastracci L, Fiocca R. Lung fibrosis : an undervalued finding in COVID-19 pathological series. Lancet Infect Dis 2021; 21:e72.

15. Gentile F, Aimo A, Forfori F, Clemente A, Cademartiri F. COVID-19 and risk of pulmonary fibrosis : the importance of planning ahead. 2020; .

16. Udwadia ZF, Koul PA, Richeldi L. Post - COVID lung fibrosis : The tsunami that will follow the earthquake. $2021 ; 41-47$.

17. Chaudhary S, Natt B, Bime C, Knox KS, Glassberg MK. Antifibrotics in COVID-19 Lung Disease : Let Us Stay Focused. 2020; 7:1-5.

18. Garcia-revilla J, Deierborg T, Venero JL, Boza-serrano A, Garcia-revilla J, Boza-serrano A. Hyperinflammation and Fibrosis in Severe COVID-19 Patients : Galectin-3, a Target Molecule to Consider. 2020; 11:1-6.

19. Antoniou K, Markopoulou K, Tzouvelekis A, Trachalaki A, Vasarmidi E, Organtzis J, Tzilas V, Bouros E, Kounti G, Rampiadou C et al: Efficacy and safety of nintedanib in a Greek multicentre idiopathic pulmonary fibrosis registry: A retrospective, observational, cohort study. ERJ Open Res 2020; 6:00172-02019.

20. Margaritopoulos GA, Trachalaki A, Wells AU, Vasarmidi E, Bibaki E, Papastratigakis G, Detorakis S TN and AK. Pirfenidone improves survival in IPF: Results from a real-life study. BMC Pulm Med 2018; 18:.

21. Wu A, Peng Y, Huang B, Ding X, Wang X, Niu P et al. Genome composition China., and divergence of the novel coronavirus (2019-nCoV) originating in. Cell Host Microbe 2020; 27:325-328.

22. Chan JF, Kok KH, Zhu Z, Chu H, To KK, Yuan S et al. Genomic characterization of the 2019 novel human-pathogenic coronavirus isolated from a patient with atypical pneumonia after visiting Wuhan. Emerg Microbes Infect 2020; 9:221-236.

23. Paules CI, Marston HD FA. Coronavirus infections - more than just the common cold. JAMA 2020; .

24. Zhou P, Yang XL, Wang XG, Hu B, Zhang L, Zhang W et al. A pneumonia outbreak associated with a new coronavirus of probable bat origin. Nature 2020; 579:270-273. 
25. Hui DS, I Azhar E, Madani TA, Ntoumi F, Kock R, Dar O et al. The continuing 2019nCoV epidemic threat of novel coronaviruses to global health - the latest 2019 novel coronavirus outbreak in Wuhan, China. Int J Infect Dis 2020; 91:264-266.

26. Chen N, Zhou M, Dong X, Qu J, Gong F, Han Y et al. Epidemiological and clinical characteristics of 99 cases of 2019 novel coronavirus pneumonia in Wuhan, China: a descriptive study. Lancet 2020; 395:507-13.

27. Wrapp D, Wang N, Corbett KS, Goldsmith JA, Hsieh CL, Abiona O et al. Cryo- EM structure of the 2019-nCoV spike in the prefusion conformation. Science (80- ) 2020; 367:1260-1263.

28. Mason RJ. Pathogenesis of COVID-19 from a cell biology perspective. Eur Respir J 2020; 55:9-11.

29. Kordzadeh-kermani E. Pathogenesis, clinical manifestations and complications of COVID-19. 2020; 15:1287-1305.

30. https://timesofindia.indiatimes.com/life-style/health-fitness/health-news/new-coronavirusimpact-on-kids-new-covid-strain-and-its-impact-on-children-all-your-questionsanswered/photostory/82043986.cms?picid=82044356. no date; .

31. Baas T, Taubenberger JK, Chong PY, Chui P KM. SARS-CoV virus-host interactions and comparative etiologies of acute respiratory distress syndrome as determined by transcriptional and cytokine profiling of formalin-fixed paraffin-embedded tissues. Interf Cytokine Res 2006; 26:399-317.

32. Wen Y, Deng BC, Zhou Y, Wang Y, Cui W, Wang W et al. Immunological features in patients with pneumonitis due to influenza A H1N1 infection. J Investig Allergol Clin Immunol 2011; 21:44-50.

33. Xu J, Xu X, Jiang L, Dua K, Hansbro PM, Liu G. SARS-CoV-2 induces transcriptional signatures in human lung epithelial cells that promote lung fibrosis. 2020; $1-12$.

34. Chuang H, Ho L, Harn H. Recent Findings on Cell-Based Therapies for COVID19Related Pulmonary Fibrosis. 2021; 30:1-4.

35. Albert RK, Smith B, Perlman CE SD. Is progression of pulmonary fibrosis due to ventilation-induced lung injury? Crit Care Med Am Thorac Soc 2019; 200:140-151.

36. Mach WJ, Thimmesch AR, Pierce JT PJ. Consequences of hyperoxia and the toxicity of oxygen in the lung. Nurs Res Pr 2011; 1-7.

37. McDonald LT. Healing after Covid-19: Are Survivors at Risk for Development of Pulmonary Fibrosis?. Am J Physiol Cell Mol Physiol 2020; .

38. Res M, Ruts C, Hospital CR, Sciences M, Committee IE, Crh-smims S. Antifibrotic drugs for idiopathic pulmonary fibrosis: What we should know? Indian J Med Res 2020; 152:177-180. 
39. Dimitroulis IA. Nintedanib: A novel therapeutic approach for idiopathic pulmonary fibrosis. Respir Care 2014; 59:1450-1455.

40. Hu Q, Noor M, Wong YF, Hylands PJ, Simmonds MSJ, Xu Q, Jiang D, Hendry BM, Xu Q. In vitro anti-fibrotic activities of herbal compounds and herbs. 2009; 3033-3041.

41. Agrawal, Pawan K., Chandan Agrawal and GB. Quercetin: Antiviral Significance and Possible COVID-19 Integrative Considerations. Nat Prod Commun 2020; 15:p.1934578X20976293.

42. Huang, F., Li, Y., Leung E et al. A review of therapeutic agents and Chinese herbal medicines against SARSCOV-2 (COVID-19). Pharmacol Res no date; 158:104929.

43. Su, H., Yao, S., Zhao, W., Li, M., Liu, J., Shang, W., ... \& Xu Y. Discovery of baicalin and baicalein as novel, natural product inhibitors of SARS-CoV-2 3CL protease in vitro. Biorxiv 2020; .

44. Hu S, Wang J, Zhang Y, Bai H, Wang C, Wang N HL. Three salvianolic acids inhibit 2019-nCoV spike pseudovirus viropexis by binding to both its RBD and receptor ACE2. J Med Virol 2021; 93:3143-3151.

45. Moghaddam, E., Teoh, BT., Sam S et al. Baicalin, a metabolite of baicalein with antiviral activity against dengue virus. Sci Rep 2014; 4:5452.

46. Chu, M., Xu, L., Zhang, M. B., Chu, Z. Y., \& Wang YD. Role of Baicalin in AntiInfluenza Virus A as a Potent Inducer of IFN-Gamma. Biomed Res Int 2015; .

47. Mahmud SMH, Akter F, Rahman S, Ahmed K, Rahman H, Chen W, Moni MA. Bioinformatics and system biology approach to identify the influences of SARS-CoV-2 infections to idiopathic pulmonary fibrosis and chronic obstructive pulmonary disease patients. Brief Bioinform 2021; 00:1-20.

48. McGroder CF, Zhang D, Choudhury MA et al. Pulmonary fibrosis 4 months after COVID-19 is associated with severity of illness and blood leucocyte telomere length. Thorax 2021; . 